\title{
Transição para a creche e bem-estar emocional dos bebês em Portugal
}

\author{
Carla Peixoto \\ Centro de Investigação e Inovação em Educação, Escola Superior de Educação, Instituto Politécnico do Porto - \\ Porto - Portugal \\ Sílvia Barros \\ Centro de Investigação e Inovação em Educação, Escola Superior de Educação, Instituto Politécnico do Porto - \\ Porto - Portugal \\ Vera Coelho \\ Faculdade de Psicologia e de Ciências da Educação, Universidade do Porto - Porto - Portugal \\ Joana Cadima \\ Faculdade de Psicologia e de Ciências da Educação, Universidade do Porto - Porto - Portugal \\ Ana Isabel Pinto \\ Faculdade de Psicologia e de Ciências da Educação, Universidade do Porto - Porto - Portugal \\ Manuela Pessanha \\ Escola Superior de Educação, Instituto Politécnico do Porto - Porto - Portugal
}

\begin{abstract}
Resumo
O presente estudo analisa a relação entre a implementação de práticas de transição do contexto familiar para a creche e o bem-estar emocional dos bebês durante o primeiro de mês de frequência neste contexto extrafamiliar. Foram recolhidos dados, antes e após a entrada do bebê na creche, junto das mães e das educadoras de 90 bebês.Os resultados indicaram que o número de práticas de transição reportadas pelas educadoras se revela positivamente associado ao bem-estar emocional dos bebês durante o primeiro mês de frequência da creche. Estes resultados recomendam a implementação de um conjunto diversificado de práticas de transição, antes e após a entrada na creche, no sentido de facilitar a adaptação dos bebês ao novo contexto desenvolvimental.
\end{abstract}

Palavras-chave: Creche; bebês; bem-estar da criança.

\section{Transition to childcare and infants' well-being in Portugal}

\begin{abstract}
The present study analyzes the relationship between the implementation of transition practices from home to day care center and the emotional wellbeing of the infants during the first month of attendance in this extrafamiliar environment. Data were collected, before and after the infant entered the day care center, with the mothers and teachers of 90 infants. The results indicated that the number of transition practices reported by teachers is positively associated with the emotional well-being of infants during the first month of day care attendance. These results recommend the implementation of a diversified set of transition practices, before and after entry into the day care center, to facilitate the infants' adaptation to the new developmental setting. Keywords: Child day care; infants; child welfare.
\end{abstract}

\section{Transición a la guardería y bienestar emocional de los bebés en Portugal}

\section{Resumen}

En el presente estudio se analiza la relación entre la implementación de prácticas de transición del contexto familiar a la guardería y el bienestar emocional de los bebés, durante el primer mes de frecuencia de este contexto extra-familiar. Se recolectaron datos, antes y tras la entrada del bebé en la guardería, por intermedio de las madres y de las educadoras de 90 bebés. Los resultados indicaron que el número de prácticas de transición reportadas por las educadoras se revela positivamente asociado al bienestar emocional de los bebés durante el primer mes de frecuencia a la guardería. Estos resultados recomiendan la implementación de un conjunto diversificado de prácticas de transición, antes y tras la entrada en la guardería, en el sentido de facilitar la adaptación de los bebés al nuevo contexto de desarrollo.

Palabras clave: Jardines infantiles; bebés; bienestar del nino. 


\section{Introdução}

A creche representa um importante contexto de cuidados e de educação para muitos bebês e crianças. A transição do contexto familiar para a creche pode ser desafiante para as crianças, as suas famílias e as instituições que as acolhem (e.g., Balaban, 2011; Merril, 2010; Rapoport \& Piccinini, 2001). Para os bebês, em particular, a entrada na creche constitui um desafio desenvolvimental considerável, uma vez que implica o estabelecimento de relações com novos adultos num contexto extrafamiliar, numa fase em que ainda se encontram aconstruir relações de vinculação no contexto familiar (Daniel \& Shapiro, 1996). Os estudos acerca desta transição, em particular durante o primeiro ano de vida, são ainda escassos, especialmente em Portugal, pelo que, nesta investigação, se pretende compreender as práticas de transição do ambiente familiar para a creche e a sua associação com o bem-estar emocional do bebê.

Em Portugal, a creche é uma das respostas sociais mais utilizadas pelas famílias com crianças em idade inferior a 3 anos. Em 2013, a taxa de cobertura média das creches e amas oficiais era de $46.2 \%$ (Portugal Continental), tendo ocorrido nos últimos anos mudanças nos seus horários e períodos de funcionamento (Equipa de Estudos e Políticas, s.d.) que se ajustam melhor às necessidades das famílias. No nosso país, há uma elevada percentagem de crianças com ambos os pais a trabalharem a tempo inteiro $(60 \%)$ e cerca de $76 \%$ de mulheres com filhos até 3 anos de idade estão integradas no mercado de trabalho, uma das taxas mais elevadas da União Europeia (Organization for Economic Co-Operation and Development [OECD], 2011).

Considerando o elevado número de famílias que recorre aos serviços de creche para os bebês, a forma como se organiza a transição inicial do contexto familiar para a creche e como os bebês experienciam essa transição assumem-se como relevantes, à luz do modelo bioecológico do desenvolvimento humano (e.g., Bronfenbrenner \& Morris, 1998). De acordo com este modelo os contextos do desenvolvimento humano encontram-se organizados numa hierarquia de sistemas interligados, de progressiva complexidade e abrangência (e.g., Bronfenbrenner \& Morris, 1998), enfatizando a importância de se considerarem os vários contextos que influenciam e são influenciados pela criança, as transações entre esses sistemas, assim como a consistência e a continuidade das práticas nos contextos de vida da criança (Bairrão, 1995). Neste sentido, a riqueza social do mesossistema, definido pelas relações existentes entre dois ou mais contextos nos quais a criança é participante ativa (e.g., família, creche), deriva da frequência e da qualidade dessas relações (Garbarino \& Ganzel, 2000), podendo contribuir para que a adaptação da criança à creche seja bem-sucedida.

Ao entrarem na creche, os bebês são confrontados com um conjunto de mudanças que vão além da separação da família, nomeadamente mudanças a nível do espaço e da sua organização, das rotinas e das pessoas com quem interagem (Datler, Ereky-Stevens, Hover-Reisner, \& Malmberg,
2012). Reconhecendo a importância de uma adaptação bem-sucedida à creche, vários autores e entidades com responsabilidades na área da educação (e.g., Balaban, 2011; O'Connor, 2013; Segurança Social, 2010) recomendam a planificação cuidadosa da transição do contexto familiar para a creche. Peixoto e cols. (2014) sistematizam um conjunto de práticas de transição, organizando-as em dois momentos: antes e após a entrada do bebê na creche. Relativamente ao período anterior à entrada na creche, apresentam exemplos de práticas, como: conhecer o bebê e a família (e.g., rotinas, interesses); fornecer informação relevante oralmente e/ou por escrito sobre o funcionamento da instituição; convidar a família para conhecer a sala e recomendar o aumento gradual da permanência do bebê na sala, assim como o uso de objetos de transição. Entre as práticas a serem adotadas durante as primeiras semanas de frequência da creche, encontram-se: os bebês podem utilizar o(s) seu(s) objeto(s) de conforto preferido(s); são incorporadas nas rotinas da sala as rotinas de cuidados desenvolvidas na família; a família tem oportunidade de telefonar ou de visitar a sala para saber como está o bebê; a família recebe informação diariamente sobre a forma como está a adaptação do bebê e é questionada sobre a forma como lida com este processo.

Apesar do reconhecimento da importância deste tipo de práticas, até à data, o conhecimento acerca do seu uso em creche é escasso. A maioria dos estudos acerca da transição tem-se focado na transição da educação pré-escolar para o primeiro ciclo do ensino básico, ensino fundamental no Brasil (Martinati \& Rocha, 2015). É também escassa a literatura acerca da relação entre as práticas de transição e o processo de adaptação do bebê à creche, o que justifica a pertinência de se investigar esta problemática, particularmente num período e num contexto histórico e social em que se verifica um elevado recurso a esses serviços.

No que se refere à adaptação do bebê à creche, destaca-se a falta de consenso existente na literatura relativamente à sua definição e caracterização, assim como à sua delimitação temporal (Pasinato \& Mosmann, 2015; Rapoport \& Piccinini, 2001). Num trabalho de revisão sobre a entrada e a adaptação de bebês e crianças pequenas à creche, Rapoport e Piccinini (2001) evidenciam que, para alguns autores, o período de adaptação teria início nos primeiros contatos dos pais com a creche (Vitória \& Rossetti-Ferreira, 1993 citado por Rapoport \& Piccinini, 2001), enquanto, para outros, decorreria entre o momento de ingresso da criança e (a) o fim do primeiro mês (Bloom-Feshbach, Bloom-Feshbach, \& Gaughram, 1980 citado por Rapoport \& Piccinini, 2001) ou (b) três a seis meses após o ingresso (Fein, 1995; Feinetal, 1993; Rodriguez, 1981 citados por Rapoport \& Piccinini, 2001). Segundo Portugal (1998), a adaptação à creche implica o modo como a criança experiencia este contexto e como a sua organização é integrada e transformada nas novas relações. Essa autora destaca que a adaptação não abrange apenas os comportamentos observados no período inicial, mas também os comportamentos que a criança expressa ao longo do tempo. Por sua vez, Skouteris, McNaught e Dissanayake (2007) operacionalizam a avaliação da 
adaptação das crianças à creche, focandoo estado afetivo da criança. Propõem avaliar aadaptação através da forma como as crianças se sentem na chegada à creche, durante o dia, à saída da creche e no tempo passado no contexto familiar. As autoras assumem que os indícios apresentados pela criança em relação ao seu bem-estar emocional são indicadores da forma como ela adapta-se às mudanças decorrentes da entrada na creche.

O conceito de bem-estar, inicialmente associado à saúde e ao estado físico, é atualmente definido de forma mais abrangente, sendo relativamente consensual que este conceito engloba também o estado emocional e social dos indivíduos. De acordo com Dodge, Daly, Huyton e Sanders (2012), o bem-estar pode ser afetado por vários acontecimentos de vida ou situações desafiantes. Promover o bem-estar das crianças é um objetivo comum a todos os que com elas interagem e assumem um papel ativo na promoção do seu desenvolvimento e educação. A legislação de vários países estabelece, inclusivamente, que as creches devem criar um ambiente saudável e estimulante e que promova o bem-estar emocional das crianças (Bagdi \& Vacca 2005; European Commission Employment \& Social Affairs and Equal Opportunities DG, 2011). O foco no bem-estar, particularmente nos sentimentos negativos e positivos da criança, fornece um "feedback" importante ao cuidador da criança, ajudando-o a identificar o que necessita ser alterado na prestação de cuidados e o que parece estar a funcionar positivamente.

Enquadrado no âmbito do projeto de investigação mais amplo "Transição dos bebês para a creche: comunicação família-creche, qualidade dos contextos e adaptação do bebê" (FCOMP010124FEDER029509; FCT PTDC/MHCCED/4007/2012), cuja finalidade é estudar a transição dos bebês do contexto familiar para a creche durante o primeiro ano de vida, este estudo tem por objetivo analisar o processo de transição de bebês para a creche, especificamente a relação entre a implementação de práticas de transição do contexto familiar para a creche e o bem-estar emocional de bebês durante o primeiro mês de frequência da creche.

\section{Método}

\section{Participantes}

Participaram neste estudo mães e educadoras (auxiliares de ação educativa ou educadoras de infância) de 90 bebês que frequentavam a creche. Todas as creches da grande área metropolitana do Porto, Portugal, foram contactadas seguindo uma ordem aleatória, tendo-se verificado que 232 tinham berçário. Destas, foram incluídas no estudo as primeiras 90 creches que tinham bebês inscritos para iniciar a frequência do berçário entre setembro de 2013 e março de 2014 e que aceitaram participar. Foi pedido que, em cada creche, fosse selecionada aleatoriamente uma família dentre as que tinham pré-inscrito o/a filho/a para iniciar a frequência do berçário quando o bebê tivesse entre 4 e 9 meses de idade e que fosse indicado o profissional de educação que passava mais tempo na sala com os bebês. $O$ estudo foi apresentado às creches e às famílias e foi obtido o consentimento informado. Os procedimentos foram aprovados pela Comissão Nacional de Proteção de Dados.

As mães tinham, em média, 30 anos de idade $(D P=$ 3.98) e 14.33 anos de escolaridade $(D P=3.55)$; o rendimento familiar médio era de $1622.40 €(D P=670.77)$. Os bebês tinham idades compreendidas entre os 4 e os 10 meses $(M=6.0, D P=1.34)$ e distribuíam-se equitativamente em termos de gênero. Os profissionais participantes, todos do gênero feminino, tinham, em média, 42.53 anos de idade $(D P=9.97)$ e 11.10 anos de escolaridade $(D P=3.64)$, sendo 7 educadoras de infância e 83 auxiliares de educação. Em média, a sua experiência em contexto de creche, era de 8.36 anos $(D P=6.50)$.

\section{Instrumentos}

Questionário sobre práticas de transição dos bebês para a creche-QPTBC (Peixoto, Pinto, \& Barros, 2013). O QPTBC foi desenvolvido com o objetivo de recolher informação sobre a implementação de práticas de transição dos bebês do contexto familiar para a creche e as ideias dos profissionais acerca da sua importância. Para a sua construção, realizou-se uma revisão da literatura, analisaram-se outros instrumentos de avaliação (e.g., NCEDL, 1996) e foram consideradas as publicações da Segurança Social (2010) relativas à creche. Procedeu-se a um estudo piloto, no qual participaram profissionais de educação (com e sem formação superior) com experiência em creche, e que não faziam parte da amostra do estudo. A versão final do QPTBC inclui uma lista de 25 práticas de transição, tendo sido solicitado aos profissionais que assinalassem as práticas realizadas na creche onde trabalham e que indicassem o que pensam sobre cada uma delas (não é necessária, é uma boa ideia ou é uma boa ideia, mas existem constrangimentos à sua implementação). Este estudo apresenta apenas os resultados relativos à implementação de práticas de transição. De forma a obter uma pontuação global relativa à variável Práticas de transição, foi contabilizado o número de práticas implementadas $(\alpha=0.69)$.

Questionário de Experiência na Creche - DEQ (Skouteris \& Dissanayake, 2001). O DEQ avalia o processo de adaptação do bebê à creche. Foi traduzido e adaptado para português por Cadima, Pinto e Coelho (2013), após autorização dos autores e seguindo as fases recomendadas (e.g., Sireci, Yang, Harter, \& Ehrlich, 2006). Inclui questões relativas ao bem-estar emocional da criança (e.g., felicidade da criança ao chegar à creche, ao ver o educador, ao longo do dia na creche e no momento de ir embora), à manutenção das rotinas do contexto familiar na creche, à adaptação global da criança à creche e à satisfação da família com o processo de transição. Neste trabalho foi utilizada apenas a média dos dados obtidos nos cinco itens sobre o bem- 
-estar emocional do bebê na primeira e quarta semanasde frequência da creche, avaliados pelas mães ( $\alpha=0.89$ e $\alpha=$ 0.81 , respetivamente) e pelas educadoras ( $\alpha=0.83$ e $\alpha=$ 0.89 , respetivamente) numa escala de 6 pontos ( $1=$ quase nunca, 6 = quase sempre).

Horas na creche. O número de horas que os bebês passavam na creche, por dia, durante a primeira e quarta semanas de frequência da creche, foi registado, numa tabela, pelas mães e pelas educadoras, tendo sido calculado o número médio de horas para cada semana.

Questionário sobre características sociodemográficas da família (Pessanha, Barros, Pinto, \& Cadima, 2013). Utilizou-se este questionário para obter informação sobre as características da família (e.g., agregado familiar, rendimento familiar, anos de escolaridade, habilitações literárias e situação profissional dos pais).

The Home Observation for Measurement of the Environment Inventory - HOME (Caldwell \& Bradley, 1984, 1997). Para avaliar a qualidade do ambiente familiar, utilizou-se a versão portuguesa da HOME para crianças entre os 0 e os 2 anos de idade, composta por 45 itens dicotómicos ( 0 = não; $1=\operatorname{sim}$ ), organizados em seis subescalas: (a) responsividade verbal e emocional dos pais, (b) aceitação dos comportamentos da criança, (c) organização do ambiente físico e do tempo da criança, (d) disponibilização de materiais de aprendizagem adequados, (e) envolvimento dos pais com a criança, e (f) oportunidade para experimentar variedade na estimulação diária. Foi utilizada a pontuação global (i.e., soma de todos os itens; $\alpha=0.69$ ).

Infant Behavior Questionnaire - Revised - IBQ-R (Rothbart \& Gartstein, 2013). O IBQ-R permite avaliar o temperamento do bebê. Vários estudos têm relatado bons indicadores de validade, adequação e consistência interna desta medida (e.g., Klein, Putnam, \& Linhares, 2009). Garts- tein e Rothbart (2003) propõem a constituição de três fatores - Extroversão, Afeto negativo e Regulação -, para análise dos dados obtidos através do questionário. No presente estudo foram apenas utilizados os dados do fator Afeto negativo $(\alpha=0.85)$.

Questionário sobre características estruturais - Berçário - QSCE-B (Barros, Pessanha, Pinto, \& Cadima, 2013). Esta medida foi utilizada para recolher dados sobre características sociodemográficas dos profissionais (e.g., idade, escolaridade, anos de experiência profissional) e características das suas salas.

\section{Procedimento}

Foi realizada uma visita domiciliária às famílias, antes dos bebês iniciarem a frequência da creche, para cotar a HOME (com base em entrevista e observação direta das interações e do ambiente físico da casa da família) e para preencher o IBQ-R. As visitas tiveram uma duração média de 2 horas. O QPTC foi preenchido sob o formato de entrevista, entre a segunda e a quarta semanas de frequência do bebê junto do profissional que passava mais tempo na sala com os bebês. Os dados relativos ao bem-estar emocional dos bebês (DEQ) e ao número de horas de frequência da creche foram recolhidos especificamente no final da primeira e quarta semanas de frequência da creche. Tanto as mães quanto as educadoras preencheram autonomamente o DEQ.

\section{Resultados}

A Tabela 1 apresenta as estatísticas descritivas de todas as variáveis analisadas no presente estudo. As educa-

Tabela 1. Estatísticas descritivas das variáveis em estudo.

\begin{tabular}{lccc}
\hline & $M$ & $D P$ & Min. - Max. \\
\hline P. de transição & 20.44 & 2.84 & $12.00-25.00$ \\
BEES1 (mães) & 4.99 & 0.95 & $1.40-6.00$ \\
BEE S4 (mães) & 5.30 & 0.78 & $2.50-6.00$ \\
BEE S1 (educadoras) & 5.00 & 0.89 & $2.60-6.00$ \\
BEE S4 (educadoras) & 5.54 & 0.62 & $3.00-6.00$ \\
Variáveis concomitantes & & & $1.55-5.98$ \\
Afeto negativo do bebê & 3.37 & 0.82 & $23.00-42.00$ \\
Qualidade do AF & 32.82 & 4.16 & $107.00-293.00$ \\
Idade do bebê à entrada na creche (dias) & 179.53 & 38.92 & $2.10-11.00$ \\
Horas/creche S1 & 6.54 & 2.08 & $3.30-10.70$ \\
Horas/creche S4 & 7.71 & 1.51 & \\
\hline
\end{tabular}

Nota. P. de transição = Número de práticas de transição. BEE = Bem-estar emocional. Qualidade do AF = Qualidade do ambiente familiar. 
Tabela 2. Correlações Momento Produto de Pearson entre as variáveis em estudo.

\begin{tabular}{|c|c|c|c|c|c|c|c|c|c|c|}
\hline & 1. & 2. & 3. & 4. & 5. & 6. & 7. & 8. & 9. & 10. \\
\hline \multicolumn{11}{|l|}{ 1. P. de transição } \\
\hline 2. BEE S1 (mães) & $.27^{*}$ & & & & & & & & & \\
\hline 3. BEES4 (mães) & .17 & $.57^{* *}$ & & & & & & & & \\
\hline 4. BEES1 (ed.) & $.43^{\star *}$ & $.57^{\star *}$ & $.40^{* *}$ & & & & & & & \\
\hline 5. BEES4 (ed.) & $.23^{*}$ & $.43^{* *}$ & $.48^{* *}$ & $.43^{* *}$ & & & & & & \\
\hline 6. Sexo (bebê) & .00 & -.16 & .03 & -.03 & .02 & & & & & \\
\hline 7. Idade (bebê) & -.02 & -.14 & -.16 & -.08 & -.19 & -.05 & & & & \\
\hline 8. Afeto negativo & .03 & -.08 & -.01 & -.11 & -.01 & -.02 & -.02 & & & \\
\hline 9. Qualidade do AF & .12 & -.18 & -.05 & -.04 & .01 & -.03 & .13 & -.13 & & \\
\hline 10. Horas/creche S1 & $-.26^{*}$ & .06 & .09 & .00 & $-.23^{*}$ & .03 & .07 & -.08 & -.12 & \\
\hline 11. Horas/creche S4 & -.07 & .04 & -.04 & -.03 & -.13 & .09 & .08 & .17 & -.20 & $.59^{* *}$ \\
\hline
\end{tabular}

Nota. ${ }^{*} p<.05 .{ }^{* *} p<.01$.

doras reportaram implementar, em média, 20.44 práticas de transição, variando entre 12 e 25 . Considerando que este valor poderia variar entre 0 e 25 , estes resultados sugerem o uso de um conjunto diversificado de práticas de transição recomendadas na literatura. No que se refere ao bem-estar emocional do bebê, de acordo com as perceções das mães e das educadoras, os bebês demonstraram mais bem-estar na quarta semana do que na primeira semana de frequência. Testes $t d e$ Student para amostras emparelhadas permitiram verificar que estas diferenças são estatisticamente significativas, tanto no que se refere aos relatos das mães, $t(81)=-3.01, p=0.004$, como dos educadores, $t(81)=-5.55, p=0.000$.

Importa destacar, igualmente, que os bebês estiveram em média 6.54 horas na creche na primeira semana e 7.71 na quarta. É notória a variabilidade no que se refere ao tempo de permanência neste contexto extrafamiliar. Salienta-se o número elevado de horas de permanência da creche logo na primeira semana.

Para compreendermos a relação entre o uso de práticas de transição e o bem-estar emocional do bebê ao longo do primeiro mês de frequência da creche, primeiramente, analisamos as associações existentes entre a variável preditora de interesse (número de práticas de transição) e as variáveis critério (bem-estar emocional do bebê na primeira semana e na quarta semana - versão pais e versão educa- dores) através do cálculo do coeficiente de correlação Momento Produto de Pearson (ver Tabela 2). Incluímos também um grupo de possíveis variáveis concomitantes (afeto negativo, qualidade do ambiente familiar, sexo, idade do bebê à entrada na creche, número de horas na creche na primeira e quarta semanas).

Encontraram-se associações positivas e estatisticamente significativas entre o número de práticas de transição implementadas e o bem-estar emocional do bebê, exceto no que se refere ao relato materno na quarta semana. Verificou-se ainda a existência de uma associação negativa e estatisticamente significativa entre o número de práticas de transição e o número de horas na creche na primeira semana.

Controlando um conjunto de variáveis concomitantes, realizamos quatro análises de regressão múltipla hierárquica, uma para cada uma das variáveis critério. Optou-se por não incluir na equação de regressão as variáveis sexo e idade das crianças, uma vez que o tamanho da amostra do presente estudo implicava prudência no número de variáveis a incluir na equação e os resultados encontrados a nível da análise de correlação sugeriram associações fracas com as variáveis dependentes.

Relativamente ao bem-estar emocional do bebê na primeira semana de frequência da creche, percecionado pelas mães e pelas educadoras, o modelo de regressão total 
Tabela 3. Sumário da análise de regressão múltipla hierárquica para as variáveis que influenciam o bem-estar emocional do bebê na primeira semana.

\begin{tabular}{|c|c|c|c|c|c|c|}
\hline & \multicolumn{6}{|c|}{ Bem-estar emocional do bebê - S1 } \\
\hline & \multicolumn{3}{|c|}{ Versão mães } & \multicolumn{3}{|c|}{ Versão educadoras } \\
\hline & $B$ & SE B & $\mathrm{b}$ & $B$ & SE B & b \\
\hline Bloco 1 & \multicolumn{4}{|c|}{$R^{2}=.03$} & \multicolumn{2}{|r|}{$R^{2}=.03$} \\
\hline Afeto negativo & -0.08 & 0.12 & -.07 & -0.17 & 0.12 & -.16 \\
\hline Qualidade do AF & -0.04 & 0.03 & -.16 & -0.01 & 0.02 & -.03 \\
\hline Horas/creche S1 & 0.02 & 0.05 & .03 & -0.01 & 0.05 & -.02 \\
\hline Bloco 2 & \multicolumn{4}{|c|}{$\Delta R^{2}=.09^{* *}$} & \multicolumn{2}{|r|}{$\Delta R^{2}=.16^{\star \star *}$} \\
\hline Afeto negativo & -0.09 & 0.12 & -.08 & -0.18 & 0.11 & -.17 \\
\hline Qualidade do AF & -0.04 & 0.02 & $-.20^{+}$ & -0.02 & 0.02 & -.08 \\
\hline Horas/creche S1 & 0.05 & 0.05 & .11 & 0.04 & 0.05 & .09 \\
\hline P. de transição & 0.10 & 0.04 & $.30^{* *}$ & 0.13 & 0.03 & $.42^{\star \star \star}$ \\
\hline \multirow{2}{*}{ Modelo total } & \multicolumn{3}{|c|}{$R^{2}$ Total $=.12$} & \multicolumn{3}{|c|}{$R^{2}$ Total $=.19$} \\
\hline & \multicolumn{3}{|c|}{$R^{2}$ Ajustado $=.07$} & \multicolumn{3}{|c|}{$R^{2}$ Ajustado $=.14$} \\
\hline
\end{tabular}

Nota. ${ }^{+} p<.10 .{ }^{* *} p<.01 .{ }^{* * *} p<.001$.

Tabela 4. Sumário da análise de regressão múltipla hierárquica para as variáveis que influenciam o bem-estar emocional do bebê na quarta semana.

\begin{tabular}{|c|c|c|c|c|c|c|}
\hline & \multicolumn{6}{|c|}{ Bem-estar emocional do bebê - S4 } \\
\hline & \multicolumn{3}{|c|}{ Versão mães } & \multicolumn{3}{|c|}{ Versão educadoras } \\
\hline & $B$ & SE B & $b$ & $B$ & SE B & $\mathrm{b}$ \\
\hline Bloco 1 & \multicolumn{4}{|c|}{$R^{2}=.01$} & \multicolumn{2}{|r|}{$R^{2}=.02$} \\
\hline Afeto negativo & 0.00 & 0.11 & .00 & 0.02 & 0.09 & .03 \\
\hline Qualidade do AF & -0.01 & 0.02 & -.06 & -0.00 & 0.02 & -.01 \\
\hline Horas/ creche S4 & -0.03 & 0.06 & -.05 & -0.06 & 0.05 & -.14 \\
\hline Bloco 2 & \multicolumn{4}{|c|}{$\Delta R^{2}=.04^{+}$} & \multicolumn{2}{|r|}{$\Delta R^{2}=.07^{*}$} \\
\hline Afeto negativo & 0.00 & 0.11 & .00 & 0.02 & 0.09 & .02 \\
\hline Qualidade do AF & -0.02 & 0.02 & -.08 & -0.01 & 0.02 & -.04 \\
\hline Horas/creche S4 & -0.02 & 0.06 & -.04 & -0.05 & 0.05 & -.13 \\
\hline P. de transição & 0.06 & 0.03 & $.20^{+}$ & 0.06 & 0.02 & $.26^{*}$ \\
\hline \multirow{2}{*}{ Modelo total } & \multicolumn{3}{|c|}{$R^{2}$ Total $=.04$} & \multicolumn{3}{|c|}{$R^{2}$ Total $=.09$} \\
\hline & \multicolumn{3}{|c|}{$R^{2}$ Ajustado $=-.01$} & \multicolumn{3}{|c|}{$R^{2}$ Ajustado $=.04$} \\
\hline
\end{tabular}

Nota. ${ }^{+} p<.10 .{ }^{*} p<.05$. 
explica $12 \%$ e $19 \%$, respetivamente (Tabela 3 ). Contrariamente ao verificado no Bloco 1, o Bloco 2 contribuiu de forma estatisticamente significativa para ambas medidas de bem-estar emocional do bebê. Depois de controlar variáveis como o afeto negativo do bebê, a qualidade do ambiente familiar e o número de horas passadas na creche durante a primeira semana, o número de práticas de transição surgiu como um preditor estatisticamente significativo do bem-estar emocional do bebê na primeira semana de frequência à creche.

O modelo de regressão total explica 4\% e 9\% do bem-estar emocional do bebê na quarta semana de frequência da creche, tal como percecionado pelas mães e pelos educadores, respetivamente (Tabela 4). Apenas o Bloco 2 contribuiu de forma estatisticamente significativa para as medidas de bem-estar emocional do bebê, ainda que apenas marginalmente na medida obtida junto das mães. À semelhança dos resultados obtidos para a primeira semana, depois de controlar o afeto negativo do bebê, a qualidade do ambiente familiar e o número de horas passadas na creche durante a quarta semana, o número de práticas de transição reportado pelas educadoras surgiu como um preditor estatisticamente significativo do bem-estar emocional do bebê na quarta semana de frequência da creche. No caso da medida obtida junto das mães, esta associação surge apenas como marginalmente significativa. Estes resultados indicam que as crianças cujas educadoras relataram um maior número de práticas de transição implementadas tendem a demonstrar maior bem-estar emocional ao longo do primeiro mês na creche.

\section{Discussão}

O presente trabalho teve por objetivo estudar o processo de transição de bebês para a creche, analisando especificamente a relação entre a implementação de práticas de transição do contexto familiar para a creche e o bem-estar emocional de bebês durante o primeiro de mês de frequência da creche. Os resultados deste estudo sugerem que as instituições dedicam atenção à adaptação dos bebês ao contexto de creche, na medida em que as educadoras participantes relataram a implementação de um conjunto elevado e diverso de práticas de transição recomendadas na literatura (e.g., Balaban, 2011; O'Connor, 2013), tanto no período que antecede a entrada do bebê, como durante as primeiras semanas de frequência à creche. Dados mais específicos relativamente às várias práticas de transição adotadas por este grupo de participantes podem ser encontrados em Peixoto e cols. (2014), sendo importante destacar as seguintes práticas no período que antecede a entrada dos bebês na creche: as profissionais fornecem informação relevante sobre o funcionamento da instituição, procuram conhecer o bebê (e.g., rotinas, interesses) e a respetiva família, convidam a família a conhecer a sala que o bebê vai frequentar e recomendam o uso de objetos de transição. Durante as primeiras semanas de frequência da creche, salientam-se as seguintes práticas de transição: a entrada dos bebês é realizada de acordo com uma calendarização préestabelecida e acordada com a família; a família é incentivada a aumentar o tempo de permanência do bebê na sala de forma gradual; a família tem a oportunidade de telefonar ou visitar a sala para saber como está o bebê; a família é informada diariamente sobre o processo de adaptação do bebê e é questionada sobre como está a lidar com este processo (Peixoto \& cols., 2014).

Apesar da preocupação com uma transição bem preparada e gradual verificada no presente estudo, o número de horas que os bebês permanecem na creche logo na primeira semana é manifestamente elevado. Note-se que uma das práticas recomendadas por várias entidades, e que tende a ser das mais valorizadas por profissionais de educação de infância, é o incentivo a que a família aumente o tempo de permanência do bebê na sala de forma gradual (Peixoto \& cols., 2014). Apesar de haver um aumento no tempo médio de permanência da primeira para a quarta semana, não parece haver uma gradação suficientemente relevante ao longo da primeira semana.

No presente estudo, foi também possível verificar que o bem-estar emocional dos bebês, reportado pelas mães e pelas educadoras, aumentou ao longo do tempo. Como referido anteriormente, a literatura tem indicado a entrada na creche como um período de transição crítico e complexo para os bebês (e.g., Balaban, 2011; Merril, 2010), que envolve diversas mudanças, nomeadamente em termos de espaço, rotinas e cuidadores (Datler \& cols., 2012). Os nossos resultados sugerem que os bebês se adaptam progressivamente ao contexto extrafamiliar de educação e cuidados, revelando um maior bem-estar emocional ao longo do tempo.

Adicionalmente, os resultados do presente estudo indicaram que o número de práticas de transição implementadas constitui uma variável de particular interesse para compreender a adaptação do bebê à creche. Especificamente, o número de práticas de transição reportadas pelas educadoras parece contribuir positivamente, ainda que de uma forma modesta, para o bem-estar emocional dos bebês durante o primeiro mês de frequência da creche. O número de práticas de transição manteve o seu efeito ao longo do tempo (i.e., da primeira semana para a quarta semana) no bem-estar emocional do bebê, apesar da redução substancial verificada no seu valor preditivo. É necessário salientar que foi controlada uma importante dimensão do temperamento dos bebês - o afeto negativo. Assim, evidencia-se o papel positivo que as creches, em articulação com as famílias, podem ter na adaptação dos bebês. Tal como Portugal (2000) salientou, com base num estudo com crianças dos 12 aos 24 meses, na adaptação da criança é preciso ter em conta as características da criança e do contexto familiar, mas também da creche. Efetivamente, em geral, estes resultados sugerem que os bebês, cujas educadoras referiram implementar um maior número de práticas de transição, são percebidos como demonstrando maior bem-estar emocional no primeiro mês de frequência da creche. 


\section{Considerações finais}

De acordo com os resultados obtidos no presente estudo, e considerando também a literatura já existente sobre esta temática, apresentada previamente (e.g., Balaban, 2011; Daniel \& Shapiro, 1996; O'Connor, 2013; Peixoto \& cols., 2014), o desenvolvimento de um conjunto diversificado de práticas que facilitem a transição dos bebês do contexto familiar para a creche deve ser incentivado. Considerando a importância destas práticas para os bebês e suas famílias, os resultados do presente estudo sugerem a necessidade de garantir que os profissionais que trabalham em creche as conheçam e saibam implementar, sendo pertinente que esta área temática seja incluída nos programas de formação de profissionais de educação de infância, permitindo o aprofundamento de conhecimentos teórico-empíricos relevantes e atualizados e de competências técnicas consideradas centrais nesta área.

A importância das práticas de transição para o processo de adaptação dos bebês evidenciada no presente estudo não surpreende, mas contribui para sustentar a sua significância teórica (e.g., Balaban, 2011; Merril, 2010). Como foi referido, não é abundante a investigação que analise especificamente a experiência de crianças com menos de 1 ano de idade no período de transição entre dois contextos de educação e cuidados, pelo que este estudo nos parece uma importante contribuição para a expansão do conhecimento nessa área específica. No entanto, note-se que uma percentagem relevante da variância nas medidas de bem-estar emocional do bebê ficou ainda por explicar, e as variáveis consideradas no presente estudo não são as únicas que influenciam a adaptação do bebê e, em particular, o seu bem-estar. Outros fatores, como a qualidade do contexto de creche, poderão ajudar a explicar uma maior quantidade de variância da forma como os bebês experienciam a transição do contexto familiar para a creche. Além disso, será importante analisar a contribuição diferencial de diferentes práticas de transição nesse processo. Admitimos a necessidade de, em estudos futuros, aprofundar essas relações e outras variáveis com efeito importante, assim como fatores associados ao contexto familiar.

Importa, agora, considerar as limitações do presente estudo. Uma das limitações refere-se à representatividade da amostra, pois, embora se tenha realizado uma seleção aleatória dos participantes, o grau de participação das creches com fins lucrativos acabou sendo reduzido. Além disso, as famílias participantes acabaram por ser bastante homogêneas relativamente às características sociodemográficas. Investigações futuras devem recorrer a uma amostra representativa da população, de forma a produzir conclusões generalizáveis. Outra limitação está relacionada com as opções metodológicas relativamente às técnicas de coleta de dados. No que concerne às práticas de transição, destacamos o recurso a um único informante (i.e., educadoras) e à técnica de auto-relato. Apesar de mais exigente do ponto de vista metodológico, consideramos importante que futuros estudos procurem incluir como informantes as famílias, além dos profissionais, e outras técnicas de coleta de dados, como a entrevista e a análise de documentos relacionados com o processo de transição dos bebês. Relativamente à adaptação do bebê à creche, é importante que futuros estudos incluam outros indicadores além do bem-estar emocional, contribuindo com dados mais abrangentes.

Apesar das limitações referidas anteriormente, consideramos que os resultados obtidos no presente estudo representam um contributo importante para a literatura sobre as transições entre contextos de desenvolvimento em idades precoces, especificamente no que concerne à relevância das práticas de transição para uma adaptação bem-sucedida dos bebês à creche.

\section{Referências}

Bagdi, A. \& Vacca, J. (2005). Supporting early childhood socialemotional wellbeing: The building blocks for early learning and school success. Early Childhood Education Journal, 33(3), 145150. doi: 10.1007/s10643-005-0038-y

Bairrão, J. (1995). A perspectiva ecológica em psicologia da Educação. Psicologia, X(3), 7-30.

Balaban, N. (2011). Transition to group care for infants, toddlers and families. Em D. M. Laverick \& M. R. Jalongo (Orgs.), Transitions to early care and education, educating the young child (pp. 7-20). New York: Springer Science-Business Media.

Barros, S., Pessanha, M., Pinto, A., \& Cadima, J. (2013). Questionário de características estruturais: Berçário. (Versão não publicada). Porto: Faculdade de Psicologia e Ciências da Educação da Universidade do Porto.

Bronfenbrenner, U. \& Morris, P. A. (1998). The ecology of developmental processes. Em W. Damon \& R. M. Lerner (Orgs.), Handbook of Child Psychology (5a ed., Vol. 1, pp. 993-1028). New York: John Wiley.

Cadima, J., Pinto, A. I., \& Coelho, V. (2013). Questionário de Experiência na Creche-DEQ. (Versão portuguesa não publicada). Porto: Faculdade de Psicologia e de Ciências da Educação da Universidade do Porto.

Caldwell, B. M. \& Bradley, R. H. (1984). Administration manual, revised edition, Home Observation for Measurement of the Environment. Little Rock: University of Arkansas.

Caldwell, B. M. \& Bradley, R. H. (1997). Escala de Avaliação do Ambiente Familiar: Versão portuguesa (Universidade do Porto, Centro de Psicologia, Trad.). Centro de Psicologia, Universidade do Porto, Portugal (Trabalhooriginal publicado em 1984).

Daniel, J. \& Shapiro, J. (1996). Infant transitions: Home to centerbased child care. Child \& Youth Care Forum, 25 (2), 111-123. 
Datler, W., Ereky-Stevens, K., Hover-Reisner, N., \& Malmberg, L-E. (2012). Toddler's transition to out-of-home day care: Settling into a new care environment. Infant Behavior and Development, 35 (3), 439-451.

Dodge, R., Daly, A., Huyton, J., \& Sanders, L. (2012). The challenge of defining wellbeing, International Journal of Wellbeing, 2(3), 222235. doi:10.5502/ijw.v2i3.4

Equipa de Estudos e Políticas (s.d.). Carta Social. Rede de serviços e equipamentos. Relatório de 2013. Gabinete de Estratégia e Planeamento. Recuperado: 3 de fev. 2016. Disponível: http:// www.cartasocial.pt/pdf/csocial2013.pdf

European Commission Employment \& Social Affairs and Equal Opportunities DG. (2011). Child well being and quality of childcare. Synthesis Report. Organized by Sandy Ruxton. European Commission.

Garbarino, J. \& Ganzel, B. (2000). The human ecology of early risk. Em J. Shonkoff \& S. Meisels (Orgs.), Handbook of early childhood intervention ( $2^{\mathrm{a}}$ ed., pp. 76-93). New York: Cambridge Press.

Gartstein, M. A. \& Rothbart, M. K. (2003). Studying infant temperament via the revised infant behavior questionnaire. Infant Behavior and Development, 26(1), 64-86. doi:10.1016/S0163-6383(02)00169-8

Klein, C., Putnam, S. P., \& Linhares, M. B. (2009). Assessment of temperament in children: Translation of instruments to portuguese (Brazil) language. Interamerican Journal of Psychology, 43(3), 552-557.

Martinati, A. Z. \& Rocha, M. S. P. M. L. (2015). "Faz de conta que as crianças já cresceram": o processo de transição da Educação Infantil para o Ensino Fundamental. Psicologia Escolar e Educacional, 19 (2), 309-319.

Merril, S. (2010). Child Care: It's a transition for parents too! Young Children, 65 (5), 60-61.

National Center for Early Development and Learning [NCEDL] (1996). Transition practices survey. Chapel Hill: University of North Carolina at Chapel Hill.

O'Connor, A. (2013). Understanding transitions in the early years: Supporting change through attachment and resilience. New York: Routledge.

Organization for Economic Co-Operation and Development [OECD] (2011). Doing better for families. Portugal. Recuperado: 5 de fev. de 2016. Disponível: http://www.oecd.org/portugal/47704295.pdf

Pasinato, L. \& Mosmann, C. P. (2015). Coparentalidade em genitores de bebês com indicativos de dificuldades de inserção escolar. Psicologia Escolar e Educacional, 19 (1), 31-40. DOI: 10.1590/2175-3539/2015/0191791
Peixoto, C., Coelho, V., Pinto, A. I., Cadima, J., Barros, S., \& Pessanha, M.(2014). Transição de bebés do contexto familiar para a creche: práticas e ideias dos profissionais. Sensos-e, 1(2). Recuperado: 14 de mar. 2016. Disponível: http://sensos-e.ese.ipp.pt/?p=6599

Peixoto, C., Pinto, A. I., \& Barros, S. (2013).Questionário sobre práticas de transição dos bebés para a creche. (Versão não publicada). Porto: Faculdade de Psicologia e de Ciências da Educação da Universidade do Porto.

Pessanha, M., Barros, S., Pinto, A. I., \& Cadima, J. (2013). Questionário sobre características sociodemográficas da família (Questionário não publicado). Porto: Instituto Politécnico do Porto, Universidade do Porto, Portugal.

Portugal, G. (1998). Crianças, famílias e creches. Uma abordagem ecológica da adaptação do bebé à creche. Lisboa: Porto Editora.

Portugal, G. (2000). Subsídios para a compreensão das inter-relações criança-família-creche. Em A. M. Fontaine (Org.), Parceria famíliaescola e desenvolvimento da criança. Partenariado familia-escuela y desarrollo de los niños. Porto: Edições ASA.

Rapoport, A. \& Piccinini, C. A. (2001). Concepções de educadoras sobre a adaptação de bebês à creche. Psicologia: Teoria e Pesquisa, 17 (1), 69-78.

Rothbart, M. K. \& Gartstein, M. A. (2013). Infant Behavior Questionnaire - Revised (Tradução original para português do Brasil por Klein \& Linhares, 2006, adaptado para português europeu por M. Pessanha, V. Coelho, \& S. Barros, 2013).

Segurança Social (Org.) (2010). Manual de Processos-Chave para a Creche ( $2^{\mathrm{a}}$ edição). Recuperado: 16 de set. 2014. Disponível: http://www4.seg-social.pt/documents/10152/13337/gqrs_creche_ processos-chave

Skouteris, H., McNaught, S., \& Dissanayake, C. (2007). Mothers' transition back to work and infants' transition to child care: Does work-based child care make a difference?. Child Care in Practice,13(1), 33-47.doi:10.1080/13575270601103432

Skouteris, H. \& Dissanayake, C. (2001). Daycare experience questionnaire. (Manuscrito não publicado). Bundoora, Australia: La Trobe University.

Sireci, S. G., Yang, Y., Harter, J., \& Ehrlich, E. J. (2006). Evaluating guidelines for test adaptations: A methodological analysis of translation quality. Journal of Cross-Cultural Psychology, 37(5), 557-567. doi:10.1177/0022022106290478

Recebido em: 21 de junho de 2016 Aprovado em: 17 de março de 2017 


\section{Sobre as autoras}

Carla Peixoto (cpeixoto@ese.ipp.pt)

Doutora em Psicologia. Assistente Convidada. Escola Superior de Educação, Instituto Politécnico do Porto. Porto, Portugal; Professora Auxiliar Convidada. Instituto Universitário da Maia. Maia, Portugal.

Sílvia Barros (silviabarros@ese.ipp.pt)

Doutora em Psicologia. Professora Adjunta. Escola Superior de Educação, Instituto Politécnico do Porto. Porto.

Vera Coelho (veracoelho_up@hotmail.com)

Doutoranda em Psicologia. Faculdade de Psicologia e de Ciências da Educação, Universidade do Porto. Porto.

Joana Cadima (joana.cadima@gmail.com)

Doutora em Psicologia. Investigadora. Faculdade de Psicologia e de Ciências da Educação, Universidade do Porto. Porto.

Ana Isabel Pinto (ana@fpce.up.pt)

Doutora em Psicologia. Professora Auxiliar. Faculdade de Psicologia e de Ciências da Educação, Universidade do Porto. Porto.

Manuela Pessanha (pessanha@ese.ipp.pt)

Doutora em Psicologia. Professora Adjunta. Escola Superior de Educação, Instituto Politécnico do Porto. Porto.

Este trabalho é financiado por Fundos FEDER através do Programa Operacional Factores de Competitividade - COMPETE e por Fundos Nacionais através da FCT - Fundação para a Ciência e a Tecnologia no âmbito do projeto FCOMP-01-0124-FEDER-029509; FCT - PTDC/MHCCED/4007/2012. 